\title{
Practical Tips for Modelling Lot-Sizing and Scheduling Problems
}

\author{
Waldemar Kaczmarczyk ${ }^{\star}$
}

\begin{abstract}
This paper presents some important alternatives for modelling Lot-Sizing and Scheduling Problems. First, the accuracy of models can improved by using short time buckets, which allow more detailed planning but lead to higher computational effort. Next, valid inequalities make the models tighter but increase their size. Sometimes it is possible to find a good balance between the size and tightness of a model by limiting a priori the number of valid inequalities. Finally, a special normalization of the variables simplifies the presentation of results and validation of models.
\end{abstract}

Keywords: production, lot-sizing and scheduling, mixed integer programming

Mathematics Subject Classification: 90B30, 90C11

Revised: 5 December 2009

\section{INTRODUCTION}

This paper considers various mixed-integer programming (MIP) models of lot-sizing and scheduling problems, for several products with deterministic, dynamic demand, on machines with limited capacity. Increasing the capabilities of computers and MIP solvers increases the possibility of applying standard MIP methods to solve real planning problems. It is however necessary to describe them using appropriate model formulations (Belvaux and Wolsey, 2001).

Below, two models are discussed, the Capacitated Lot Sizing Problem with Linked lots (CLSPL) proposed by Suerie and Stadtler (2003) and the Proportional Lot-sizing and Scheduling Problem (PLSP) proposed by Haase (1994), see also Drexl and Haase (1995). The CLSPL belongs to the class of large bucket problems, which allow many set-up operations within a single period.

The PLSP is a small bucket problem, i.e. it allows only one set-up operation within a single period. It allows the processing of two products in a single period, one before and one after the set-up operation. Small bucket models allow the sequence of products to be controlled in contrast to the CLSPL. This is an important advantage, e.g. in the case of sequence-dependent set-up costs or times.

\footnotetext{
* Department of Operations Research and Information Technology, AGH University of Science and Technology, Kraków, Poland. E-mail: waldek@agh.edu.pl
} 
In general, large bucket models are more appropriate for planning problems with long periods and small bucket models for problems with short periods. There exists however a grey area where both types of model may be applied.

Independent of the choice of model, the period length must be chosen. Usually that choice is limited, i.e. one may choose periods equal to a week, day or shift. There is also the possibility of splitting real periods (macro-periods) into several fictitious micro-periods. Small periods allow more detailed planning, among others more detailed accounting of inventory holding costs, but lead to models with a larger number of variables which are harder to solve. In this paper, the trade-off between accuracy and complexity of models resulting from the choice of period length is studied.

Micro-periods are mentioned in several papers (Fleischmann, 1990; Fleischmann, 1994; Drexl and Kimms, 1997) but their number has not been a subject of research yet. Suerie (2005) compared results of the CLSPL and PLSP models but only for an identical number of periods.

Adding valid inequalities is one of the methods used to make MIP models easier to solve (Belvaux and Wolsey, 2001; Wolsey, 2002; Pochet and Wolsey, 2006). The number of all possible valid inequalities may however be very high. Therefore it is often not practical to add all the valid inequalities a priori to the model (Pochet and Wolsey, 2006; Belvaux and Wolsey, 2001, p. 101).

In such cases, there are three options. Firstly, one may rely only on the capabilities of solvers to generate various cuts, which is straightforward, but does not always ensure short computation times. Next, one may write one's own separation procedure, to choose inequalities which should be added to the model at a given node of the branch and cut method. This is a really challenging task, but is most likely to yield an efficient solving procedure. Finally, one may a priori limit the number of inequalities. The last option is easy to apply and will be presented in this paper.

Visualization of solutions and validation of models are seldom subjects of discussion. In this paper, a special normalization of parameters and variables is presented which makes them much easier visualize for a wide class of problems.

The next section presents data parameters, variables and both discussed models. Section 3 presents a trade-off driven by the size of time buckets. Section 4 shows computational results for various numbers of valid inequalities. Section 5 presents the normalization of parameters and variables. Finally, Section 6 gives conclusions.

\section{MODELS}

The dynamic lot-sizing problem, also called the Wagner-Whitin problem (Wagner and Whitin, 1958), assumes a discrete planning horizon, i.e. time is modelled as a finite number of time points. The intervals between the time points are called time periods or time buckets. Demand is given per period and varies over time. All changes in the system are assumed to occur at the time points themselves. A discrete-time model can be employed to approximate a continuous-time system but in many cases it describes reality better than continuous time.

In this paper, models are discussed which adopt the dynamic lot-sizing problem for the case with many products and a limited capacity of resources. They are 
usually called lot-sizing and scheduling problems (Drexl and Kimms, 1997; Jans and Degraeve, 2008).

Parameters and variables common to both models discussed in this paper are presented in Tables 1 and 2 .

Table 1. Parameters

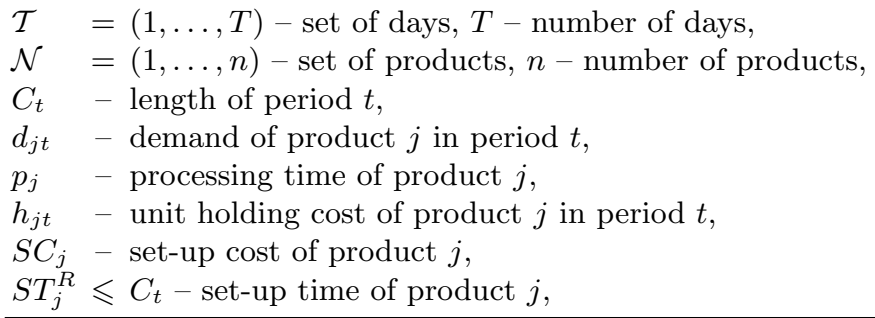

Table 2. Variables

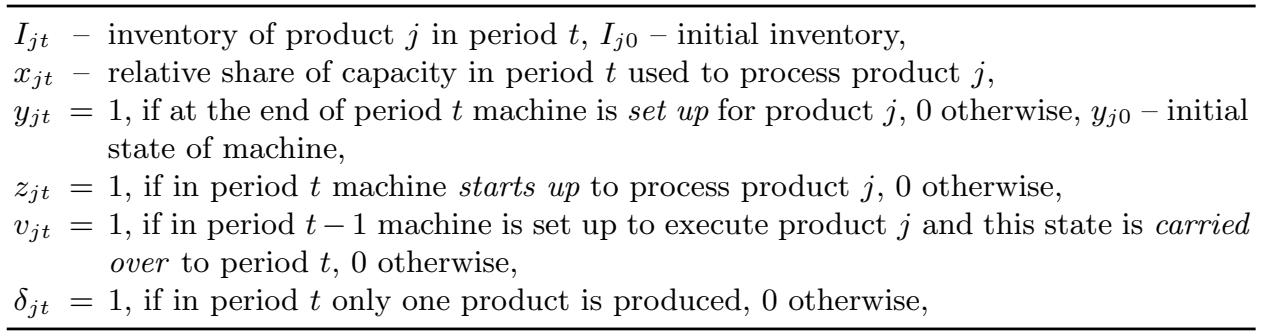

In all models described in this paper, all variables with period index $t \leqslant 0$ are assumed to be equal to 0 . There are only two exceptions. First, the inventory variables $I_{j 0}$ represent non-zero initial inventories. Second, the set-up state variables $y_{j 0}$ define the initial state of the machine.

\subsection{THE CAPACITATED LOT SIZING PROBLEM}

The basic large bucket model is called the Capacitated Lot Sizing Problem (CLSP). It allows many set-up operations in a single period but does not take into account the possibility of set-up carry-over between periods. One may save set-up costs if some product $j$ is processed at the end of a period $t$ and then, at the beginning of the next period $t+1$, the same product $j$ may be produced without additional set-up operation. This is called set-up carry over or lot-linking. Sometimes it is even possible to carry over the same state to two consecutive periods, i.e. from period $t-1$ to $t$ and $t+1$, and to process only a single product in period $t$.

Various models have been developed which allow set-up carry-over (Haase, 1994; Haase, 1996; Sox and Gao, 1999; Suerie and Stadtler, 2003). Below a model is presented that was proposed by Suerie and Stadtler (2003) called the CLSP with Linked lots (CLSPL). 
A mixed-integer programming model of the CLSPL can be stated as follows:

$$
\begin{array}{rlrl}
\min \sum_{t \in \mathcal{T}} \sum_{j \in \mathcal{N}}\left(S C_{j} z_{j t}+\right. & \left.h_{j t} I_{j t}\right) \\
I_{j t-1}+x_{j t}-d_{j t}=I_{j t}, & & t \in \mathcal{T}, j \in \mathcal{N} \\
p_{j} x_{j t}+S T_{j}^{R} z_{j t} \leqslant C_{t} y_{j t}, & & t \in \mathcal{T}, j \in \mathcal{N} \\
\sum_{j \in \mathcal{N}}\left(p_{j} x_{j t}+S T_{j}^{R} z_{j t}\right) \leqslant C_{t}, & & t \in \mathcal{T} \\
\sum_{j \in \mathcal{N}} v_{j t} \leqslant 1, & & t \in \mathcal{T}, \\
v_{j t} \leqslant y_{j, t-1}, & & t \in \mathcal{T}, j \in \mathcal{N} \\
z_{j t}+v_{j t}=y_{j t}, & & t \in \mathcal{T} \\
v_{j, t-1}+v_{j t} \leqslant \delta_{j t}+1, & & t \in \mathcal{T} \backslash\{1\}, j \in \mathcal{N} \\
z_{j t}+\delta_{t} \geqslant 1, & & t \in \mathcal{T}, j \in \mathcal{N} \\
I_{j t}, x_{j t} \geqslant 0, & & t \in \mathcal{T}, j \in \mathcal{N} \\
v_{j t} \in[0,1], & t \in \mathcal{T}, j \in \mathcal{N} \\
y_{j t}, z_{j t}, \delta_{t} \in\{0,1\}, & t \in \mathcal{T}, j \in \mathcal{N}
\end{array}
$$

The objective function (1.1) represents the total set-up and inventory costs. Constraint (1.2) describes the inventory, production and demand balance. Constraint (1.3) allows non-zero production volumes only if the machine is set up for that product. Constraint (1.4) ensures that the workload does not exceed capacity. Constraint (1.5) ensures that the set-up of at most one product may be carried over from the previous period. According to (1.6), the set-up of a product may be carried over from period $t-1$ to period $t$ if the machine was set up to process that product in period $t-1$. Equality (1.7) declares that the set-up state may be either carried over from the previous period or started up in the current one. Constraint (1.8) ensures that two consecutive carry-overs of the same product $j$, i.e. from period $t-1$ to $t$ and $t+1$, are allowed only if in the middle period $t$ only one product is processed. Constraint (1.9) ensures that either a single product is processed during the whole period or some product is started up in that period.

In the original model proposed by Suerie and Stadtler (2003) the set-up variable $y_{j t}$ is replaced by the sum of the carry-over and start-up variables, i.e. $z_{j t}+v_{j t}$, which makes constraint (1.7) superfluous.

\subsection{THE PROPORTIONAL LOT-SIZING AND SCHEDULING PROBLEM}

The small bucket models allow at most one set-up operation per period. The Proportional Lot-sizing and Scheduling Problem (PLSP) is the most flexible and most 
accurate, because it allows to process two products within a single period, first before and second after the set-up operation. A mixed-integer programming model of the PLSP can be stated as follows:

$$
\begin{aligned}
& \min \sum_{t \in \mathcal{T}} \sum_{j \in \mathcal{N}}\left(S C_{j} z_{j t}+h_{j t} I_{j t}\right) \\
& I_{j t-1}+x_{j t}-d_{j t}=I_{j t}, \quad t \in \mathcal{T}, j \in \mathcal{N} \\
& p_{j} x_{j t}+S T_{j}^{R} z_{j t} \leqslant C_{t}\left(y_{j t-1}+y_{j t}\right), \quad t \in \mathcal{T}, j \in \mathcal{N} \\
& \sum_{j \in \mathcal{N}}\left(p_{j} x_{j t}+S T_{j}^{R} z_{j t}\right) \leqslant C_{t}, \quad t \in \mathcal{T} \\
& \sum_{j \in \mathcal{N}} y_{j t}=1, \quad t \in \mathcal{T} \\
& y_{j t}-y_{j, t-1} \leqslant z_{j t}, \quad t \in \mathcal{T}, j \in \mathcal{N} \\
& I_{j t}, x_{j t} \geqslant 0, \quad t \in \mathcal{T}, j \in \mathcal{N} \\
& z_{j t} \in[0,1], \quad t \in \mathcal{T}, j \in \mathcal{N} \\
& y_{j t} \in\{0,1\}, \quad t \in \mathcal{T}, j \in \mathcal{N}
\end{aligned}
$$

The objective function (2.1) represents the total set-up and inventory costs. Constraint (2.2) describes the balance of inventory, production and demand. Constraint (2.3) allows production to take values higher than zero only if the machine is set up to process a given product in the current or previous period. Inequality (2.4) ensures that total production and set-up operations do not exceed period capacity. Constraint (2.5) ensures that in every period, the set-up state variable of only one product takes the value one, i.e. the machine is set-up to process exactly one product. Equality (2.6) ensures that start-up variables take the value one if the machine is set up to process a given product in the current period but not in the previous period. The start-up variables do not have to be binary, because their values are minimized and constraint (2.6) defines their lower bound which is equal either to 1 or 0 .

In the PLSP, following valid inequalities can be applied (Belvaux and Wolsey, 2001; Wolsey, 2002; Pochet and Wolsey, 2006):

$$
\begin{array}{cl}
z_{j t} \leqslant y_{j t}, & t \in \mathcal{T}, j \in \mathcal{N} \\
z_{j t} \leqslant 1-y_{j, t-1}, & t \in \mathcal{T}, j \in \mathcal{N} \\
I_{j, t-1} \geqslant \sum_{s=t}^{t+p} d_{j s}\left[1-y_{j, t-1}-\sum_{r=t}^{s} z_{j r}\right], & t \in \mathcal{T}, p=0, \ldots, T-t, j \in \mathcal{N}
\end{array}
$$

Constraint (2.10) resets start-up variables in periods without set-up. Inequality (2.11) resets start-up variables if in the previous period the machine was already set up.

The third valid inequality (2.12) is the most important one, i.e. it has the strongest impact on computation times. This constraint determines the lower bound 
on inventory level in period $t-1$ which is necessary to satisfy demand from period $t$ to period $t+p$. The time interval $[t, t+p]$ may be interpreted as a run-out time of lots finished in period $t-1$ or earlier.

\section{SETTING OF TIME BUCKETS SIZE}

Most authors do not justify the length for assumed time buckets although that choice is difficult and important. Short periods allow more detailed and more accurate planning but lead to complex models with many variables and constraints. In this section, some aspects of the trade-off between accuracy and complexity of lot-sizing and scheduling models are discussed.

At first, let us describe a way to compare results of large and small bucket models. To solve large bucket instances with a small bucket model, i.e. to approximate a large bucket model by a small bucket model, it is necessary to prepare the demand and unit holding costs parameters in a special way.

First, the large bucket macro-periods have to be split into several micro-periods. Let us assume that there are 5 micro-periods within each macro-period. Next, the demand of every macro-period has to be assigned to its last micro-period, so that only micro-periods which are multiples of 5 have non-zero demand. Similarly, the unit holding costs have to be set equal to zero in all micro-periods with the exception of periods which are multiples of 5 .

To estimate the impact of period length on the accuracy of the PLSP model, the following experiments were executed. For several randomly generated data sets, solutions for two models were obtained, the CLSPL and PLSP with (macro-)periods subdivided into many micro-periods. The PLSP with single micro-period is identical with the model in which macro-periods are not split into micro-periods. Optimal objective values of the CLSPL served as comparative values for the PLSP.

Five data sets were randomly generated according to the following procedure. All problem instances had 12 (macro-)periods and 5 products. The demand $d_{j t}$ was drawn from the range $[10,300]$ and next with probability 0.3 set equal to 0 . To preserve the existence of a feasible solution, the demand of the first two days for all products was set equal to 0 . Processing times $p_{j}$ of all products were equal to 1 and set-up times $S T_{j}$ equal to 0 . The constant capacity of a single machine $C$ was chosen in such a way as to keep the utilization of the machine equal to $80 \%$. Unit holding costs $h_{j}$ were randomly chosen from the range $[1,5]$. The set-up costs were calculated as $S C_{j}=\lambda_{j} * h_{j} * C$, where $\lambda_{j}$ was randomly chosen from the range $[1,15]$. In this way, the set-up costs could be 1 to 15 times higher than the holding cost of the maximal single period throughput.

All instances were solved for the PLSP with 1,2 or 3 micro-periods and for set-up costs equal to $100 \%, 25 \%$ and $10 \%$ of the originally generated costs $S C_{j}$.

All tests were performed with 30 minutes time limit on an Intel Pentium IV processor with a $1.66 \mathrm{MHz}$ clock speed and 0.5 GB RAM running ILOG OPL Studio 6.0.1 (CPLEX 11) with standard settings.

In Table 3 all results are presented. For all cases, the average number of start-ups (set-up operations) increases as set-up costs decrease and as number of micro-periods 
increases. Small set-up costs make frequent set-up operations less costly and numerous micro-periods allow to schedule more start-ups.

Table 3. Average results for the CLSPL and PLSP

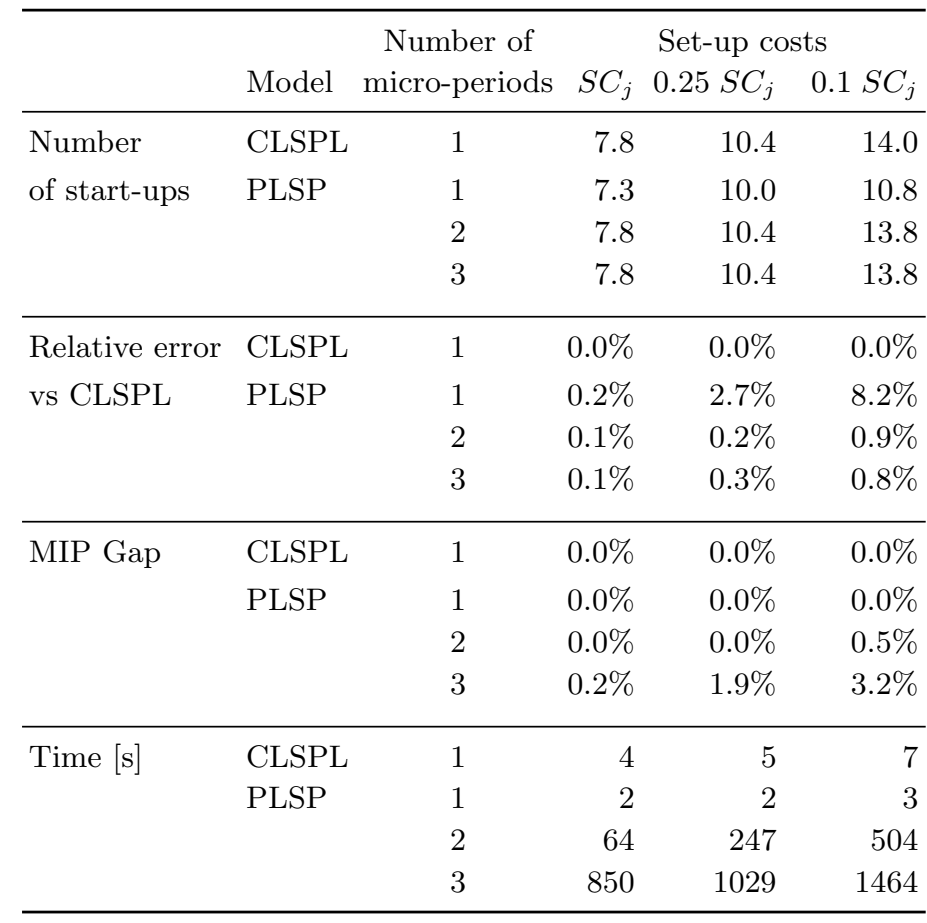

The relative error versus the $C L S P L$ was calculated as

$$
\left(f^{*}(\mathrm{PLSP}) / f^{*}(\mathrm{CLSPL})-1\right) * 100 \%
$$

where $f^{*}()$ means the best objective value found for a given model. This error is high for small a number of micro-periods and small set-up costs, in the worst case even $8.2 \%$ percent. This is easy to explain why.

A small bucket model cannot guarantee the same quality of solution as a large bucket model because it allows only one set-up operation within a period. Moreover micro-periods constitute specific additional boundaries for lot sizes and timing. Especially in the case with the smallest set-up costs and single micro-periods for which the number of start-ups in the large bucket solution is higher than the number of periods.

Already for two micro-periods, the solutions of the PLSP are close to the solutions of the CLSPL. It seems that PLSP results in a solution of good quality if the number of set-up operations is clearly smaller than the number of periods.

The MIP gap was calculated using the following formula: $\left(f^{*}(\mathrm{PLSP}) / L B-1\right) *$ $100 \%$, where $L B$ stands for the lower bound of the solution. For three micro-periods, the MIP gap is not equal to zero and the solutions could possibly be improved further. 
This may explain why for three micro-periods the objective values are worse than for two.

Finally, a decrease in set-up costs, i.e. increase of the number of start-ups in optimal solutions, and increase in the number of micro-periods radically increase the computations times.

There is an evident trade-off between the accuracy and complexity of the PLSP model driven by period length. For the data sets tested, two micro-periods would be a good compromise because the quality of the solution is already very good and the complexity much smaller than for three micro-periods.

\section{NUMBER OF VALID INEQUALITIES}

Valid inequalities are constraints which are not necessary to define a correct model but make it more tight (Belvaux and Wolsey, 2001;Wolsey, 2002; Pochet and Wolsey, 2006). Firstly, they ensure that in linear relaxations of the model, integer variables are more likely to take integer values. Secondly, they improve the lower bounds of nodes in the branch and bound algorithm. In this way, valid inequalities reduce the number of nodes needed to solve a MIP problem and may speed up execution of the branch and bound algorithm.

On the one hand, valid inequalities tighten the model, but on the other hand, they also increase its size and each iteration of the algorithm takes more time. The number of valid inequalities may grow very fast with the number of variables. Because of this, it is often not practical to add all the valid inequalities a priori to the model. In addition, only a limited number of them are really necessary to tighten the model (Pochet and Wolsey, 2006, p. 101).

Programming a special separation algorithm to choose valid inequalities which should be added to the model at given nodes of the branching algorithm is a challenging task. Commercial branch-and-cut systems are able to generate some of the valid inequalities automatically as cutting planes, but not all of them.

Finally, one may try to limit the number of valid inequalities a priori. Let us discuss this taking as example constraint (2.12). The number of such inequalities may be significantly reduced by the introduction of an upper bound $P_{\max }$ for parameter $p$ as presented below:

$$
\begin{aligned}
I_{j, t-1} \geqslant \sum_{s=t}^{t+p} d_{j s}\left[1-y_{j, t-1}-\sum_{r=t}^{s} z_{j r}\right] & \\
& \quad t \in \mathcal{T}, p=0, \ldots, \min \left\{P_{\max }-1, T-t\right\}, j \in \mathcal{N}
\end{aligned}
$$

To justify such a modification, one has to explain at first the sense of that inequality. It sets a lower bound on the inventory level at the end of period $t-1$ if in the time interval from period $t$ to period $t+p$ the machine is not set up to process given product. One may say that the parameter $p$ describes the run-out time of the inventory. In general, it may reach the end of the planning horizon, but in practice this not very likely.

To verify the impact of $P_{\max }$ on the PLSP, the following experiments were executed. Five data sets were randomly generated using a procedure identical to that 
from the previous section with a few differences. All problem instances had 30 periods. The demand $d_{j t}$ was set equal to 0 with a product-dependent probability chosen randomly from the range $[0,0.8]$. To preserve the existence of feasible solutions, the demand of the four first days for all product was set equal to 0. Set-up times $p_{j}$ were randomly chosen from the range $[60 \%, 80 \%]$ of period length.

For every data set, the PLSP has been solved with $P_{\max }$ taking values from 0 to $T$. $P_{\max }$ was always the same for all products. All tests were performed with a 5 minute time limit on an Intel Pentium IV processor with a $1.66 \mathrm{MHz}$ clock speed and 0.5 GB RAM running ILOG OPL Studio 6.0.1 (CPLEX 11) with standard settings.

In Figure 1, the results of all computational experiments are presented. The relative error describes the average relative difference between the optimal values of the objective function and the values obtained within the time limit for various $P_{\max }$ values.

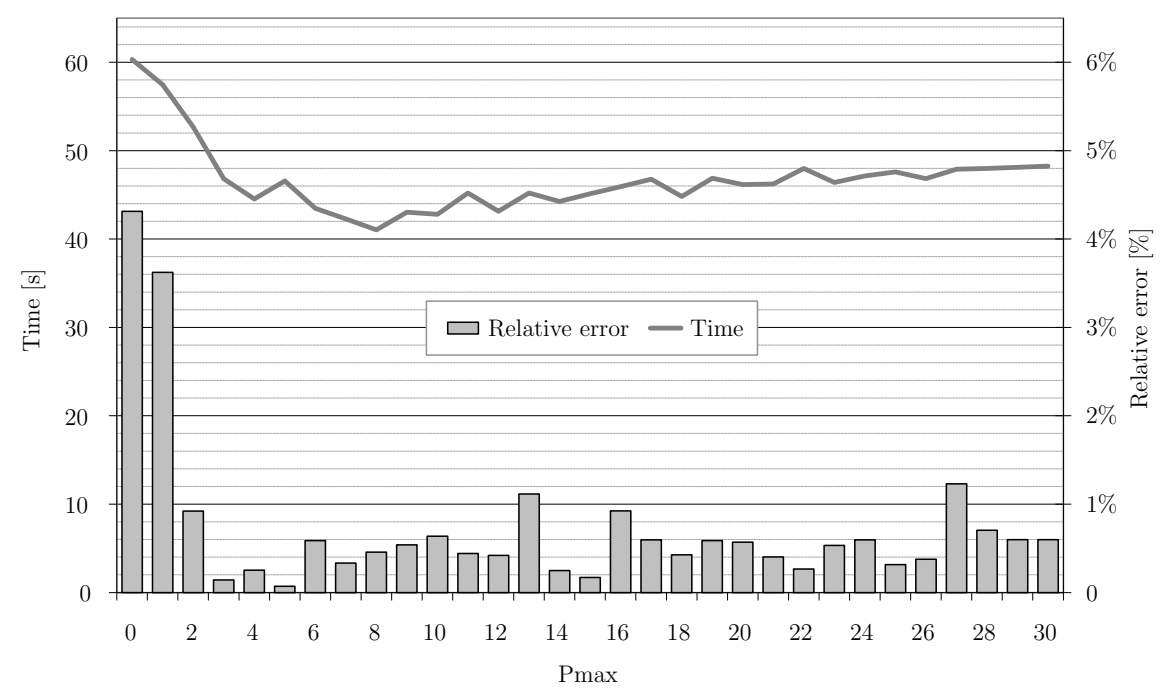

Fig. 1. Impact of number of valid inequalities

If the model does not contain any valid inequalities $(3)$, i.e. for $P_{\max }=0$, then its results are $4 \%$ worse than the optimal ones. For $P_{\max }=1$ solutions are not much better. Only $P_{\max }=2$ ensures that the distance to the optimal value is on average smaller than $1 \%$. The best objective values have been obtained for $P_{\max }=5$. The shortest average computation time was obtained for $P_{\max }=8$.

From these results, one may draw the conclusion that limiting a priori the number of valid inequalities may be a useful technique for making the lot-sizing and scheduling models easier to solve.

\section{NORMALIZATION OF VARIABLES}

Let us assume that all time buckets have the same length $C$, which is fully justified in short-term manufacturing planning and very common in computational experiments. 
In such cases, it is possible to normalize lot-sizing and scheduling models in such a way that the values of the demand, production and inventory variables are expressed in units equal to maximal throughput during a single period:

$$
T H_{j}=C / p_{j}
$$

In the normalized model several variables and parameters are replaced by their normalized versions:

$$
\begin{aligned}
S T_{j}^{\prime} & =S T_{j}^{R} / C \\
d_{j}^{\prime} & =d_{j t} / T H_{j} \\
I_{j t}^{\prime} & =I_{j t} / T H_{j} \\
x_{j t}^{\prime} & =x_{j t} / T H_{j}
\end{aligned}
$$

and two parameters, $p_{j}$ and $C$, are replaced by 1 , i.e. become equal to 1 .

In the normalized PLSP model, compared to (2), the objective function (2.1) and two constraints, (2.3) and (2.4), have different forms:

$$
\begin{gathered}
\min \sum_{t \in \mathcal{T}} \sum_{j \in \mathcal{N}}\left(S C_{j} z_{j t}+h_{j t} T H_{j} I_{j t}^{\prime}\right) \\
x_{j t}^{\prime}+S T_{j}^{\prime} z_{j t} \leqslant y_{j t-1}+y_{j t}, \quad t \in \mathcal{T}, j \in \mathcal{N} \\
\sum_{j \in \mathcal{N}}\left(x_{j t}^{\prime}+S T_{j}^{\prime} z_{j t}\right) \leqslant 1, \\
\quad t \in \mathcal{T}
\end{gathered}
$$

Moreover, the production variables $x_{j t}$ may take values only from the range $[0,1]$. The CLSPL model may be normalized in a similar way.

In Table 4 a solution of a normalized PLSP model with 4 products, 4 days and 2 shifts per day is presented. The set-up times of all products are equal to $25 \%$ of the period. Thanks to the normalization, it is easier to analyze the solution and to check various conditions. Let us look at some examples.

Normalized production variable $x_{j t}^{\prime}$ represents the relative share of period $t$ used for production of product $j$. Normalized set-up time $S T_{j}^{\prime}$ is equal to the fraction of a period necessary to set up the machine for product $j$. For example, in the third period, $60 \%$ of the capacity is utilized for production of product $1,15 \%$ for production of product 2 , and the rest is long enough to execute a single set-up operation $\left(S T_{j}^{\prime}=0.25\right)$.

The sum of the total production and set-ups times in every period has to be smaller than or equal to 1 . The total demand of a product (minus its initial inventory) is equal to the number of days necessary to satisfy it. The total workload is equal to the sum of the total production volume and number of set-up operations multiplied by normalized set-up time, i.e. $5.60+7 * 0.25=7.35$. Utilization of the machine over the whole planning horizon is equal to the total workload divided by the number of periods: $7.35 / 8 \approx 92 \%$. 
Such a normalization may also be easily adopted to the case with $m$ identical parallel machines (Kaczmarczyk, 2010). In such models, the total production during a single period takes values from the range $[0, m]$.

Table 4. An example of a normalized solution of the PLSP model

\begin{tabular}{|c|c|c|c|c|c|c|c|c|c|c|c|}
\hline & Period & 0 & 1 & 2 & 3 & 4 & 5 & 6 & 7 & 8 & \\
\hline & & Day & 1 & & 2 & 2 & 3 & 3 & 4 & & \\
\hline & Product & Shift & 1 & 2 & 1 & 2 & 1 & 2 & 1 & 2 & Total \\
\hline \multirow[t]{5}{*}{ StartUp } & 1 & & & 1 & L & & & & & 1 & 2 \\
\hline & 2 & & 1 & & & & 1 & 1 & & & 2 \\
\hline & 3 & & & & 1 & 1 & & & 1 & & 2 \\
\hline & 4 & & & & & 1 & & & & & 1 \\
\hline & Total & & 1 & 1 & 1 & 1 & 1 & & 1 & 1 & 7 \\
\hline \multirow[t]{5}{*}{ Setup } & 1 & & & 1 & L & & & & & 1 & 2 \\
\hline & 2 & & 1 & & & & 1 & 1 & & & 3 \\
\hline & 3 & & & & 1 & L & & & 1 & & 2 \\
\hline & 4 & 1 & & & & 1 & & & & & 2 \\
\hline & Total & 1 & 1 & 1 & 1 & 1 & 1 & 1 & 1 & 1 & 9 \\
\hline \multirow[t]{5}{*}{ Production } & 1 & & & 0.30 & 0.60 & & & & & 0.50 & 1.40 \\
\hline & 2 & & & 0.05 & & & & 0.95 & 0.50 & & 1.50 \\
\hline & 3 & & & & 0.15 & & & & 0.25 & 0.25 & 0.65 \\
\hline & 4 & & 0.55 & & & 0.75 & 0.75 & & & & 2.05 \\
\hline & Total & & 0.55 & 0.35 & 0.75 & 0.75 & 0.75 & 0.95 & 0.75 & 0.75 & 5.60 \\
\hline \multirow[t]{5}{*}{ Demand } & 1 & & & & & 0.45 & & 0.45 & & 0.50 & 1.40 \\
\hline & 2 & & & 0.55 & & & & 0.70 & & 0.75 & 2.00 \\
\hline & 3 & & & 0.65 & & 0.55 & & 0.45 & & 0.50 & 2.15 \\
\hline & 4 & & & 0.30 & & 0.80 & & 0.50 & & 0.45 & 2.05 \\
\hline & Total & & & 1.50 & & 1.80 & & 2.10 & & 2.20 & 7.60 \\
\hline \multirow[t]{5}{*}{ Inventory } & 1 & & & 0.30 & 0.90 & 0.45 & 0.45 & & & & 2.10 \\
\hline & 2 & 0.50 & 0.50 & & & & & 0.25 & 0.75 & & 2.00 \\
\hline & 3 & 1.50 & 1.50 & 0.85 & 1.00 & 0.45 & 0.45 & & 0.25 & & 6.00 \\
\hline & 4 & & 0.55 & 0.25 & 0.25 & 0.20 & 0.95 & 0.45 & 0.45 & & 3.10 \\
\hline & Total & 2.00 & 2.55 & 1.40 & 2.15 & 1.10 & 1.85 & 0.70 & 1.45 & & 13.20 \\
\hline
\end{tabular}

\section{CONCLUSIONS}

In this paper, three issues connected with of modelling lot-sizing and scheduling problems are presented. Firstly, the size of time buckets is discussed. The results presented show that the accuracy of small bucket models may rapidly increase with a decrease in the length of periods and with an increase in their number. Unfortunately, the complexity of models also grows very fast. Therefore, it is always necessary to find a good trade-off between accuracy and complexity. 
Next, valid inequalities are discussed which set lower bounds on inventories for all possible run-out times. The number of these inequalities may be limited by the restriction on the length of verified run-out intervals. The results presented prove that neither the minimal nor maximal length is the best choice. The best solutions or the smallest computations times are on average obtained for run-out times between 5 and 8 periods. In further research, one may evaluate more sophisticated strategies, e.g. taking into account product-specific information or demand profiles.

Finally, the normalization proposed simplifies the analysis of results and validation of models by presenting the solutions in an informative way.

\section{REFERENCES}

Belvaux, G., Wolsey, L.A. (2001). Modelling practical lot-sizing problems as mixed-integer programs, Management Science 47(7): 993-1007.

Drexl, A., Haase, K. (1995). Proportional lotsizing and scheduling, International Journal of Production Economics 40: 73-87.

Drexl, A., Kimms, A. (1997). Lot sizing and scheduling - survey and extensions, European Journal of Operational Research 99(2): 221-235.

Fleischmann, B. (1990). The discrete lot-sizing and scheduling problem, European Journal of Operational Research 44(3): 337-348.

Fleischmann, B. (1994). The discrete lot-sizing and scheduling problem with sequencedependent setup costs, European Journal of Operational Research 75(2): 395-404.

Haase, K. (1994). Lotsizing and scheduling for production planning, number 408 in Lecture Notes in Economics and Mathematical Systems, Springer-Verlag.

Haase, K. (1996). Capacitated lot sizing with linked production quantities of adjacent periods, Technical report, Working Paper 334, Institut für Betriebswirtschaftslehre, Christian-Albrechts-Universität zu Kiel, Germany.

Jans, R., Degraeve, Z. (2008). Modeling industrial lot sizing problems: a review, International Journal of Production Research 46(6): 1619-1643.

Kaczmarczyk, W. (2010). Proportional lot-sizing and scheduling problem with identical parallel machines, International Journal of Production Research. Accepted for publication.

Pochet, Y., Wolsey, L.A. (2006). Production planning by mixed integer programming, Series in Operations Research and Financial Engineering, Springer, New York.

Sox, C.R., Gao, Y. (1999). The capacitated lot sizing problem with setup carry-over, IIE Transactions 31: 173-181.

Suerie, C. (2005). Time Continuity in Discrete Time Models, Springer.

Suerie, C., Stadtler, H. (2003). The capacitated lot-sizing problem with linked lot sizes, Management Science 49 (8): 1039-1054.

Wagner, H.M., Whitin, T.M. (1958). Dynamic version of the economic lot size model, Management Science 5: 89-96.

Wolsey, L.A. (2002). Solving multi-item lot-sizing problems with an MIP solver using classification and reformulation, Management Science 48(12): 1587-1602. 